\title{
A Case Study of Text and Discourse Based on Juku, Coh-Metrix, and Linguistic Inquiry Word Count (LIWC)
}

\author{
Jia Liu \\ Foreign Languages College, Guangxi University \\ Nanning, Guangxi, China; \\ 1522796414@qq.com
}

\begin{abstract}
This paper aims to investigate the main writing features in the English time-limited compositions by third-year English major students at Guangxi University in China. Three automated assessing tools were adopted: Juku automated scoring system designed in China; Coh-Metrix automated computational evaluation tool constructed by researchers in the USA; and Linguistic Inquiry Word Count (LIWC). The results showed that there are great differences in five dimensions in the writings of high and low score compositions based on the calculation of Juku and Coh-Metrix, and there exists a strong correlation between Chinese English majors' argumentative writings and formal texts standardized by LIWC.
\end{abstract}

Keywords-Juku automated scoring system; Coh-Metrix automated computational evaluation tool; Linguistic Inquiry Word Count (LIWC)

\section{I . INTRODUCTION}

Juku is one of the automated scoring tools used by many universities in China. It was designed by Beijing Speech network technology. It can analyze vocabulary, grammar, collocations, and give an overall score to a text or discourse based on the technology of natural language processing, that of analysis of corpus, and that of education assessing. It is easy and free to use.

In China, many researchers have investigated how to use Juku to serve for the English writing teaching and learning. For example, Gu (2012) conducted an experiment to identify whether or not Juku could help students develop their writing abilities. The result of his experiment shows that the writing qualities in the experiment group improved more than the writing quality in the control group. Jiang \& Ma (2013) also conducted an experiment to test the functions of Juku that may account for the improvements in English writings. The finding was that Juku can determine the correctness of vocabulary to some extent based on the stored corpus in Juku, but it cannot judge the appropriateness of the content or semantic meanings that are off the topic. Sometimes some evident language mistakes cannot be recognized by Juku (Chen, 2011). He (2013) found that the scores given by Juku are apparently higher than those given by veteran English writing teachers.

\author{
Ying Shen \\ Foreign Languages College, Guangxi University \\ Nanning, Guangxi, China; \\ Institute of Intelligent Systems, The University of Memphis \\ Memphis, Tennessee, USA \\ shenying388@ gmail.com; sying@ memphis.edu
}

Coh-Metrix is a computational tool used to evaluate the linguistic features of a text or discourse. It was designed by the Institute of Intelligent Systems, the University of Memphis in the USA. This tool has been developed from version 1.0 to the present version 3.0. Coh-Metrix 3.0 can be used to confirm 108 indices, which can represent the cohesion of the explicit text and coherence of the mental representation of the text (McNamara \& Graesser, 2014).

Graesser et al. discuss five major factors that account for most of the variance in texts across grade levels and text categories: word concreteness, syntactic simplicity, referential cohesion, causal cohesion, and narrativity. They consider the importance of both quantitative and qualitative characteristics of texts for assigning the right text to the right student at the right time.(Graesser, McNamara, \& Kulikowich 2011)

Coh-Metrix has been used to analyze text by many researchers in China. For instance, Du \& Cai (2013) found that easability, and frequency of words, referential cohesion, and sentence length have a close relationship to the quality of writings by English major students in China. Based on the analysis of the data, they constructed a formula that can interpret $44 \%$ of highly scored English writings. This formula is: final score of a writing $=118.633-(0.778 \times$ easability $)+(0.062 \times$ word

numbers $)-(0.15 \times$ the minimum concreteness of content words).

Linguistic Inquiry Word Count (LIWC) is a text analysis software program designed to calculate the degree to which people use different categories of words across a wide array of texts (Pennebaker, Booth, and Francis, 2007). Because LIWC can demonstrate the features of vocabulary use by the calculation of the mean use of individual variables (including self-references, social words, positive emotions, negative emotions, overall cognitive words, articles and big words), it has been applied in much research concerning analyzing the characteristics of vocabulary use. (Duan, et al. 2014)

In this paper, the following research questions will be addressed:

- Are there any relationships between high and low score compositions in relation to linguistic features in the selected argumentative writings according to three 
automated scoring systems (Juku, Coh-Metrix, and LIWC)?

- What can we learn from the statistic analysis of this paper?

\section{II . RESEARCH METHODOLOGY}

\section{A. Subjects, topics, and time}

150 English compositions from 45 third-year English major students at Guangxi University were analyzed. The selected topics are confined to argumentative writings because in China argumentative writings are the main genres represented in TEM-8 (Test for English Majors Band 8). Selected subjects are students who chose the author's English academic writing class as a selective lesson during the first semester of 2014.

\section{B. Software}

Juku Automated Scoring System, Coh-Metrix Automated Computational Evaluation Tool, and Linguistic Inquiry Word Count (LIWC).

\section{Procedure}

First, 150 compositions were input into the Juku automated scoring system after the author corrected all the spelling mistakes in all the compositions; then final scores of each composition were drawn from the automated scoring system Juku.

Second, the 150 English compositions were divided into three groups according to the data from Juku: high score group (scores higher than 90), intermediate score group (scores higher than 80), and low score group. (scores higher than 70)

Third, 50 English compositions in each group were copied into Coh-Metrix automated computational evaluation tool and then 108 indices of the linguistic and discourse representations of subjects' English compositions were automatically calculated.

Fourth, 50 English compositions in each group were pasted into LIWC to draw out the data of seven variables of vocabulary use.

\section{RESULTS AND DISCUSSIONS}

- Noun overlap, adjacent sentences (binary and mean); argument overlap, adjacent sentences (binary and mean); stem overlap, adjacent sentences (binary and mean); noun overlap, all sentences (binary and mean); and argument overlap, all sentences (binary and mean) present a high relationship with high score compositions. (refer to Table 1, items 28 through 33)

- Lexical diversity demonstrated a significant relationship with the high score compositions. In other words, type- token ratio, content word lemmas; type-token ratio (all words); MTLD (all words) and VOCD (all words) affect much the quality of argumentative writings. (refer to Table 1, items 46 through 49)

- Two indices of syntactic complexity are highly related with the high score compositions. Put in other words, left embeddedness, words before main verb, and the mean number of modifiers per noun phrase are more commonly seen in high score compositions than those in low score ones. (refer to Table 1, items 67 and 68)

- Syntactic pattern density of preposition phrase density, agentless passive voice density, and gerund density are much higher in the the high score English writings than those in low score ones. In other words, preposition phrase density (incidence), agentless passive voice density (incidence), and gerund density (incidence) have the strong influence on the quality of argumentative compositions. (refer to Table 1, items 77 and 78)

- Word information of noun, verb and adjective incidences in highly scored English writings are evidently existing than in lowly scored writings. Put in other words, the number of left embeddedness, words before main verb (mean) and the number of modifiers per noun phrase (mean) are significantly more than those in low score compositions. In addition, word information of concreteness for content words (mean), imagability for content words (mean), hypernymy for verbs (mean) and hypernymy for nouns and verbs (mean) have a high relationship with high score compositions. (refer to Table 1, items 97 and 98)

- Based on Table 2, it is clearly seen that the lexical use tendency of seven variables [Self-references (I, me, my), Social words, Positive emotions, Negative emotions, Overall cognitive words, Articles (a, an, the), Big words (> 6 letters)] in the high score groups through low score groups' argumentative compositions is highly correlated with that of formal texts rather than personal texts. From this it can be considered that Chinese English majors have gained the ability to write their argumentative compositions from at least seven variables as a whole even though there exist obvious differences in using each individual variable. 
Table I : Consecutive Outcomes of 150 English Argumentative Compositions Based on Juku and Coh-Metrix

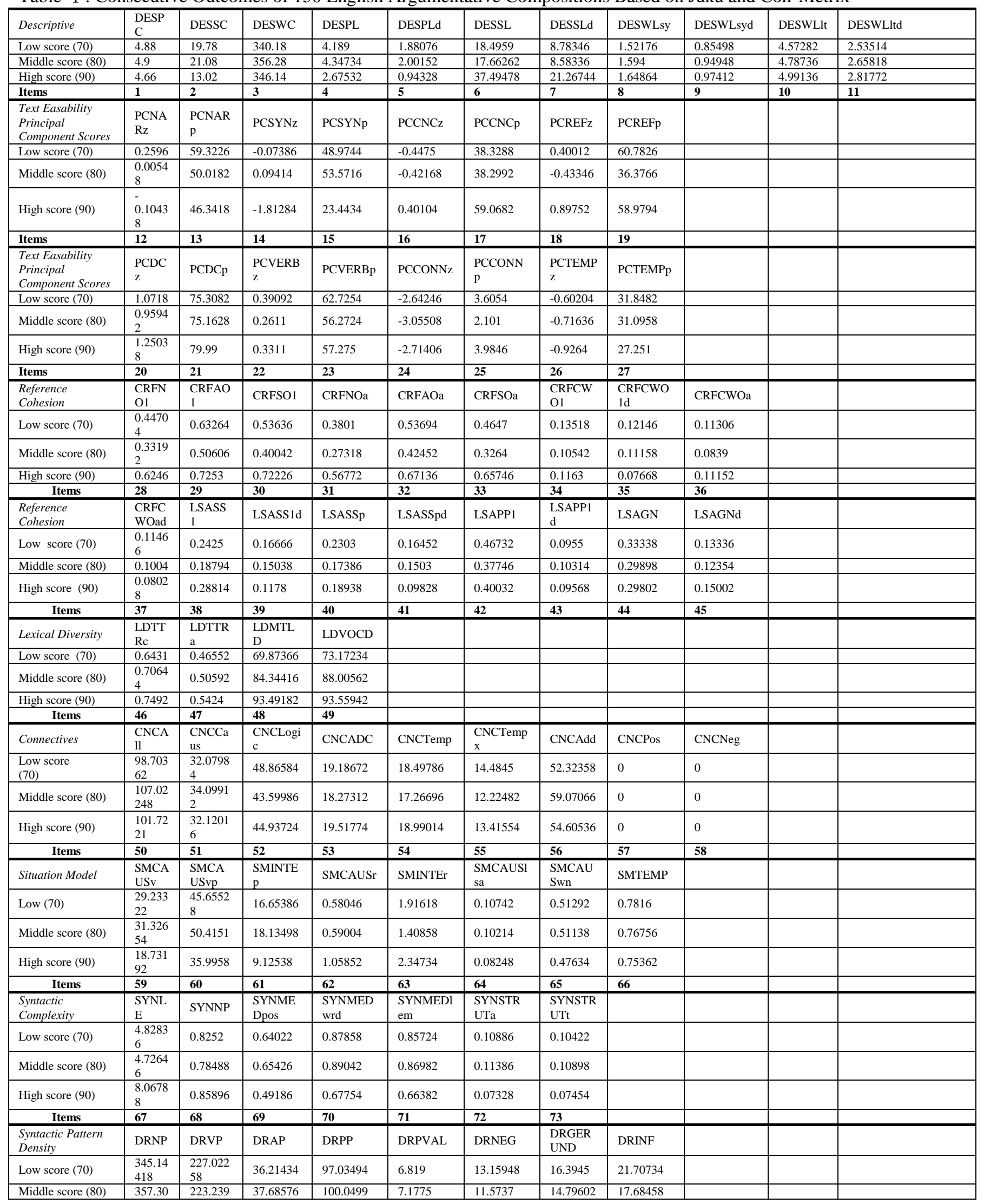




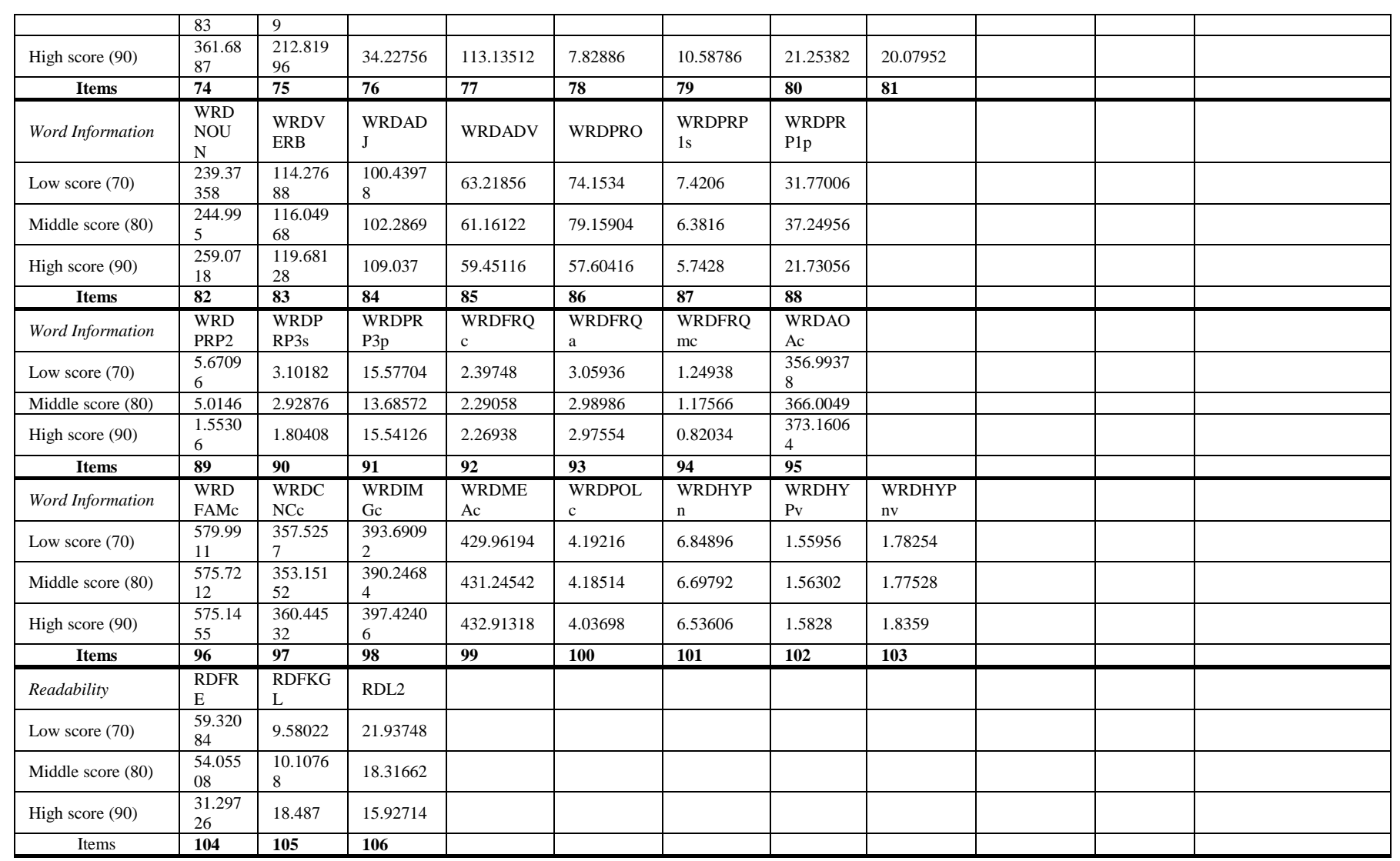

Table II : Correlations between three groups in seven variables [Self-references (I, me, my), Social words, Positive emotions, Negative emotions, Overall cognitive words, Articles (a, an, the), Big words (> 6 letters] based on LIWC

\begin{tabular}{|c|c|c|c|c|c|c|}
\hline & & High score group & Middle score group & Low score group & Personal texts & Formal texts \\
\hline \multirow[t]{3}{*}{ Middle score group } & Pearson Correlation & 1 & & & & \\
\hline & Sig. (2-tailed) & & & & & \\
\hline & variables & 7 & & & & \\
\hline \multirow[t]{3}{*}{ Middle score group } & Pearson Correlation & $.992^{* *}$ & 1 & & & \\
\hline & Sig. (2-tailed) & .000 & & & & \\
\hline & variables & 7 & 7 & & & \\
\hline \multirow[t]{3}{*}{ Low score group } & Pearson Correlation & $.982^{* *}$ & $.989^{* *}$ & 1 & . & \\
\hline & Sig. (2-tailed) & .000 & .000 & & & \\
\hline & variables & 7 & 7 & 7 & & \\
\hline \multirow[t]{3}{*}{ Personal texts } & Pearson Correlation & .646 & .721 & .709 & 1 & \\
\hline & Sig. (2-tailed) & .117 & .068 & .074 & & \\
\hline & variables & 7 & 7 & 7 & 7 & \\
\hline \multirow[t]{3}{*}{ Formal texts } & Pearson Correlation & $.989^{* *}$ & $.995^{* *}$ & $.993^{* *}$ & .718 & 1 \\
\hline & Sig. (2-tailed) & .000 & .000 & .000 & .069 & \\
\hline & variables & 7 & 7 & 7 & 7 & 7 \\
\hline
\end{tabular}

**. Correlation is significant at the 0.01 level (2-tailed). 


\section{CONCLUSIONS}

First, from the data analysis five dimensions (referential cohesion, lexical diversity, syntactic complexity, syntactic pattern density, and word formation) in a text or discourse are confirmed to exert the important role of getting a high score on argumentative compositions.

Second, during the copying of low score compositions, many spelling mistakes were obviously observed in low score compositions while this was seldom the case in high score compositions. However, because each spelling mistake was corrected before they were copied into CohMetrix, it can be inferred that the main reason of low score compositions is not necessarily vocabulary misspellings, many other reasons can be predicted.

Third, based on the LIWC outcomes, it can be concluded that English majors in China can to a great extent write formal compositions required by some necessary standards. Especially, the use of seven variables [Selfreferences (I, me, my), Social words, Positive emotions, Negative emotions, Overall cognitive words, Articles (a, an, the), Big words (> 6 letters)] in the argumentative compositions written by Chinese English majors seems equal to that of formal texts calculated by LIWC.

\section{ACKNOWLEDGMENT}

This research is financially supported by the core research project (XBS100008) of Guangxi University in 2010 and
Innovative Research Foundation for National University Students in 2014 (NO141059349).

\section{REFERENCES}

[1] A. C. Graesser, D. S. McNamara, and J.M. Kulikowich., Coh-Metrix: Providing Multilevel Analyses of Text Characteristics, Educational Researcher, vol.40, pp.223-234, 2011.

[2] C. H. Gu \& L. Wang, An empirical study of college English writing based on Juku. Journal of Yangzhou University, vol.16, no.4, pp.92-96, 2012.

[3] D.S. McNamara, A.C. Graesser, P.M. McCarthy, \& Z. Cai, Automated Evaluation of Text and Discourse with Coh-Metrix. Cambridge Press, 2014.

[4] H. Y. Du \& J. T. Cai, Expected model of accessing writing quality of Chinese English learners based on Coh-Metrix. Modern Foreign Languages, vol.3, pp.34-39, 2013.

[5] J. W. Pennebaker, R.J. Booth, and M.E. Francis. Linguistic Inquiry and Word Count: LIWC, Austin, TX: LIWC. net. 2007.

[6] X. L. He, The study of validity and reliability of Juku for the assessing of English compositions. Modern Education Technology, vol.5, pp.5559, 2013.

[7]Y. Duan et al. Linguistic style and social historical context: an automated linguistic analysis of Mao Zeddong's speeches, Proceedings of the Twenty-Seventh International Florida Artificial Intelligence Research Society Conference, pp.43-46, 2014.

[8] Y. Chen, Investigation and analysis on the application of automated essay scoring system of college English writing teaching, Chinese Journal, vol.11, pp.70-72, 2011.

[9] Y. Jiang \& W.L. Ma, Achievements or challenges? Inquiring the intelligent tutor's functions on Chinese English writings. Electronic Education Research, vol.7, pp.35-38, 2013. 